\title{
Chronic Hepatitis B Virus Infection Presenting as Crescentic Membranoproliferative Glomerulonephritis: A Distinctly Rare Occurrence
}

\author{
Nazarul Hassan Jafry, ${ }^{1}$ Abdul Saboor Khan, ${ }^{1}$ Rubina Naqvi, ${ }^{1}$ Tabassum Elahi, ${ }^{1}$ \\ Muhammed Mubarak ${ }^{2}$
}

\author{
Departments of Nephrology ${ }^{1}$ and Pathology ${ }^{2}$, \\ Sindh Institute of Urology and Transplantation (SIUT), Karachi, Pakistan
}

\begin{abstract}
Hepatitis B virus (HBV) is a highly prevalent infection worldwide. It primarily infects liver and presents with features of chronic liver disease. Rarely, it presents with extra-hepatic manifestations. Kidney involvement in HBV infection is not uncommon. However, presentation with rapidly progressive glomerulonephritis is distinctly rare. A 40-year-old man with undiscovered HBV infection presented with fever-triggered body swelling for one month. Serum creatinine was $2.3 \mathrm{mg} / \mathrm{dl}$ on admission, which increased during hospitalization to $4.5 \mathrm{mg} / \mathrm{dl}$. Kidney biopsy demonstrated crescentic membranoproliferative glomerulonephritis, immune complex mediated. Clinical, laboratory and imaging studies revealed mild chronic liver damage. Complete kidney, hepatic and virological remission was achieved with steroids, plasmapheresis and antiviral therapy. This case emphasizes on early diagnosis and institution of multimodal therapy for better outcomes.
\end{abstract}

Key words: Crescents, glomerulonephritis, hepatitis B virus, membranoproliferative glomerulonephritis, kidney biopsy.

\section{Corresponding author:}

Nazrul Hassan Jafry, MD

Department of Nephrology,

Sindh Institute of Urology and Transplantation (SIUT),

Karachi-74200, Pakistan,

Email address: dr_nazar_jafferi@yahoo.com

Received:5 August 2021 Accepted: 14 September, 2021.

PJKD 2021;5(3):82-86

Introduction:

Around 350-400 million people are infected with hepatitis B virus (HBV) globally and approximately 786,000 dies annually from HBV-related liver disease. ${ }^{1,2}$ In Pakistan, the prevalence of $\mathrm{HBV}$ infection is as high as 3-5\%. ${ }^{3}$ HBV-related glomerulonephritis (HBV-GN) is one of the extra-hepatic manifestations of chronic HBV infection and is uncommon but problematic. HBV-GN may present with mild to moderate proteinuria, hematuria and nephrotic syndrome. Rapidly progressive glomerulonephritis (RPGN) is a very infrequent presentation. Diagnosis of HBV-GN is made through a series of serological and molecular tests of HBV infection coupled with kidney biopsy.

The most common presentations of HBV-GN are membranous nephropathy (MN), membranoproliferative glomerulonephritis (MPGN), and mesangial proliferative glomerulonephritis (MesPGN). ${ }^{4}$ Although focal crescents may be seen in HBV-GN, usually superimposed on some other pattern of immune complex-mediated injury, presence of $>50 \%$ crescents on kidney biopsy (thus, qualifying for labelling as crescentic GN) is rare. Typically, MN is more common in children than adults, whereas MPGN is more common in adults. ${ }^{5}$ Various therapeutic approaches for HBV-GN have been tested, including interferon, lamivudine, and entecavir with or without corticosteroids. Some case series suggest that antiviral treatment may improve proteinuria and kidney outcome. ${ }^{6,7}$ However, most of the results are derived from small-scale case series, and a standard of care has not been established yet. 


\section{Chronic HBV \& RPGN}

We report a case of a 40-year-old man with HBV infection presenting with facial puffiness and body swelling and was diagnosed as crescentic MPGN, immune complex-mediated, on kidney biopsy. He was treated successfully with a combination of high dose immunosuppressive treatment, plasmapheresis and entecavir.

\section{Case report:}

A 40-year-old male was admitted with one month history of facial and generalized body swelling and abdominal distention preceded by fever. On physical examination, he looked ill, and his blood pressure was 135/85 $\mathrm{mmHg}$, pulse, 98/min and had edema. On systemic examination, respiratory system revealed dull percussion note with decreased breath sounds below $9^{\text {th }}$ intercostal space bilaterally and abdominal examination revealed distended abdomen with fluid thrill and shifting dullness.

His laboratory tests showed blood urea of $225 \mathrm{mg} / \mathrm{dl}$ (normal range: $10-50 \mathrm{mg} / \mathrm{dl}$ ), serum creatinine, $2.3 \mathrm{mg} / \mathrm{dl}$ (normal range: $0.5-1.5 \mathrm{mg} / \mathrm{dl}$ ) and serum albumin, $1.4 \mathrm{~g} / \mathrm{dl}$. The levels of serum aspartate aminotransferase (AST) were $48 \mathrm{U} / \mathrm{L}$ (normal range: 0-40 U/L), serum alanine aminotransferase (ALT), $66 \mathrm{U} / \mathrm{L}$ (normal range: 0-40 U/L), and urinalysis showed albuminuria $3+$ and numerous red blood cells (RBCs) and $4.2 \mathrm{~g}$ proteinuria on spot urinary protein to creatinine ratio. He had low C3, $0.07 \mathrm{~g} / \mathrm{L}$ (normal range: $0.79-1.52 \mathrm{~g} / \mathrm{L}$ ) and $\mathrm{C} 4,0.14 \mathrm{~g} / \mathrm{L}$ (normal range: $0.16-0.38 \mathrm{~g} / \mathrm{L}$ ). Antinuclear antibodies (ANA), cytoplasmic anti-neutrophil cytoplasmic antibodies (cANCA), peri-nuclear anti-cytoplasmic antibodies (p-ANCA) and anti-glomerular basement antibodies were not detected. Rheumatoid factor was positive at $38.8 \mathrm{IU} / \mathrm{ml}$ (Normal: <20 IU/ml). Ultrasonography of abdomen showed moderate ascites. Chest X-ray showed mild bilateral pleural effusion, more marked on right side (Figure 1). Viral serology was positive for hepatitis B surface antigen (HBsAg) and hepatitis B e antigen (HBeAg) (91.57 and $>1.0$, respectively) with high HBV DNA by quantitative PCR $\left(7.6 \times 10^{7}\right.$ copies $\left./ \mathrm{ml}\right)$, while anti-hepatitis C virus (HCV) was non-reactive. Assay for serum cryoglobulins was negative. Antibodies to core antigen (anti$\mathrm{HBcIgM}$ ) were negative. Ascitic fluid analysis showed transudate with total leucocyte count of 400 and neutrophils, $96 \%$ and lymphocytes, $4 \%$.

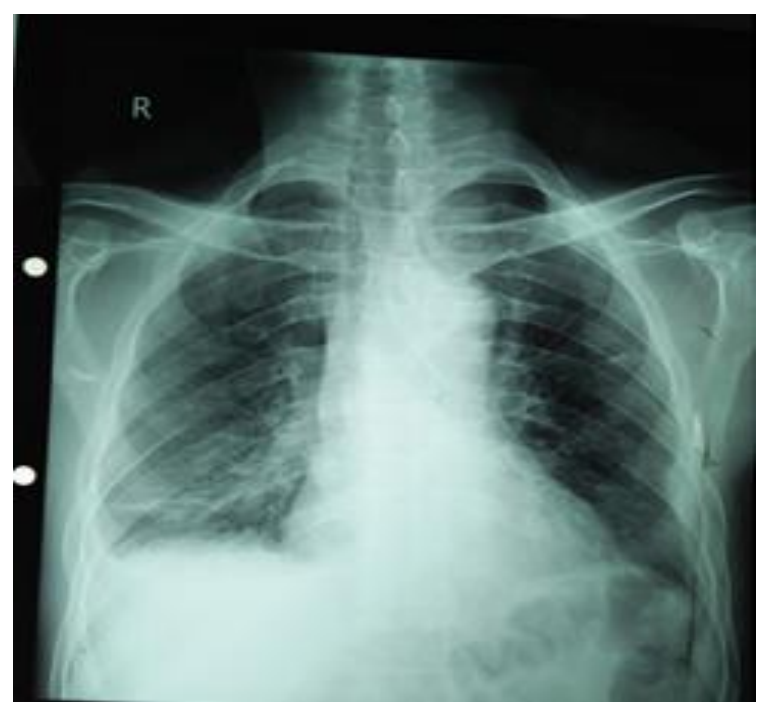

Figure 1. Chest X-ray of the patient showed bilateral mild pleural effusion, more marked on right side.

Serum creatinine increased to $4.5 \mathrm{mg} / \mathrm{dl}$ on second day of hospitalization and a provisional diagnosis of RPGN secondary to HBV infection was proposed. A percutaneous kidney biopsy was performed, which demonstrated crescentic MPGN (Figure 2). All glomeruli were abnormal and showed MPGN pattern of injury. In addition, 12 out of 18 glomeruli showed cellular crescents. There was moderate interstitial inflammation and acute tubular injury. Immunofluorescence study showed diffuse granular mesangial and membranous positivity of $\operatorname{IgG}(+++)$ and $\mathrm{C} 3(+)$, while rest of the immune reactants were negative (Figure 3).

Following biopsy results, he was started with intravenous pulse methylprednisolone (500 mg/day for 3 days) followed by oral steroids at $1 \mathrm{mg} / \mathrm{kg} / \mathrm{day}$, and plasmapheresis (14 sessions over a period of two weeks) under 


\section{Chronic HBV \& RPGN}

cover of entecavir ( $0.5 \mathrm{mg}$ every other day modified according to eGFR). He was discharged on maintenance dose of steroids and entecavir with serum creatinine of $2.1 \mathrm{mg} / \mathrm{dl}$, blood urea, $121 \mathrm{mg} / \mathrm{dl}$, blood pressure (126/82 $\mathrm{mmHg}$ ), and non-oliguria. On follow-up, his serum creatinine was $1.7 \mathrm{mg} / \mathrm{dl}$ at two weeks and $1.2 \mathrm{mg} / \mathrm{dl}$ at three months. His liver function tests were normalized, and HBV DNA became negative at 7-month follow-up.

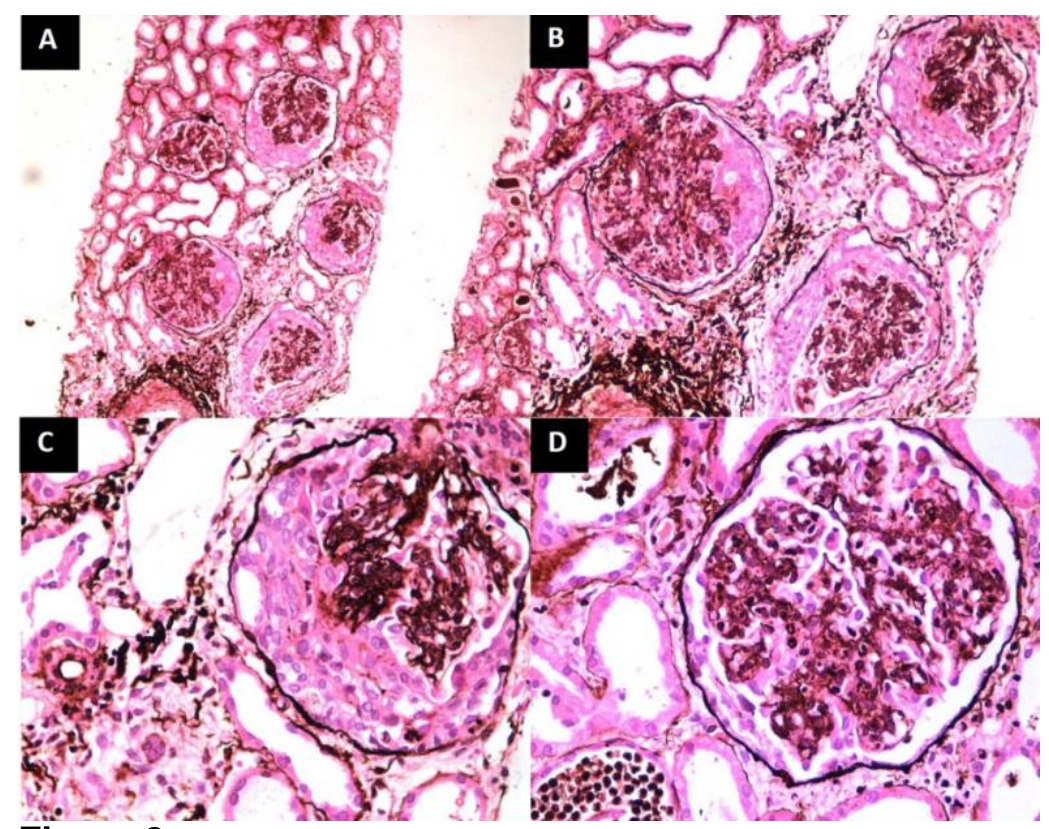

Figure 2. Light microscopic features on kidney biopsy. A. Low-power microscopic view of a representative area of kidney biopsy showing crescents in four out of five glomeruli. (Jone' s methaneamine silver, $\times 100$ ). B. Medium-power view showing cellular crescents in three glomeruli. (Jone' s methaneamine silver, $\times 200$ ). C. High-power view showing cellular crescent. The glomerular tuft of capillaries is collapsed due to extrinsic compression by the crescent. (Jone' s methaneamine silver, x400). D. High-power view showing membranoproliferative pattern of injury with marked mesangial proliferation and thickening of capillary walls by double-contouring. No crescent is seen in this glomerulus. (Jone' s methaneamine silver, $\times 400)$.

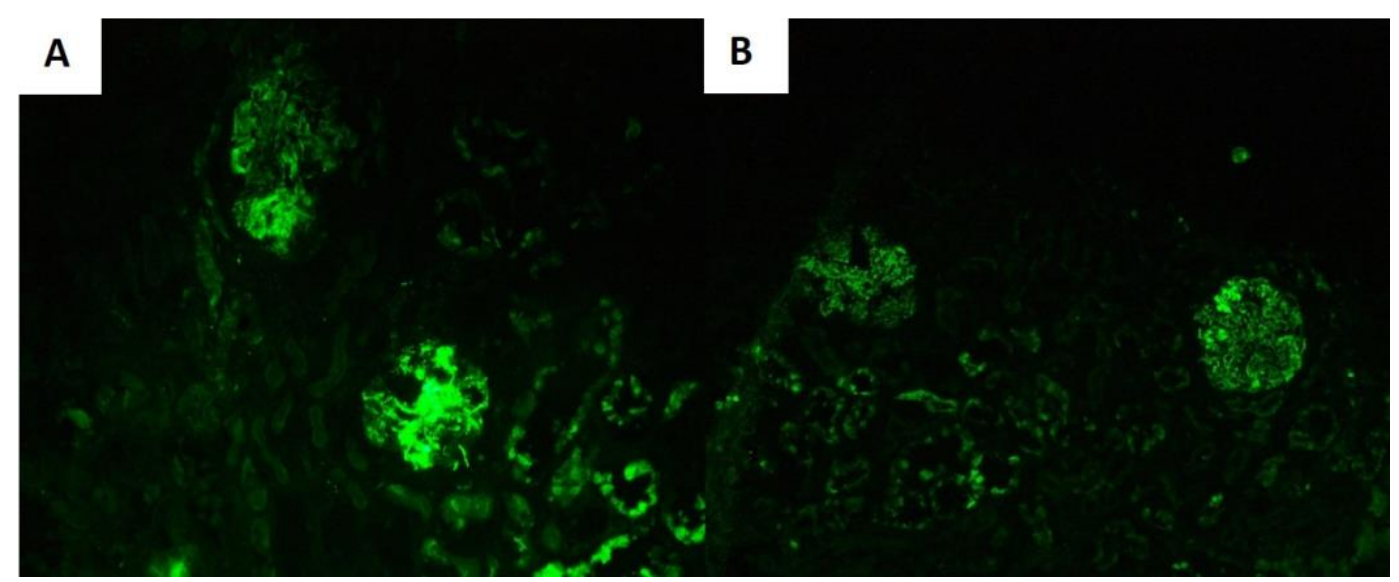

Figure 3. Immunoflourescence findings on kidney biopsy. A. Two glomeruli showing strong (3+) mesangial and membranous positivity of $\operatorname{IgG}$. B. Two glomeruli showing moderate (2+) positivity of $\mathrm{C} 3$ in the same distribution as $\operatorname{IgG}$.

\section{Discussion:}

The association of chronic HBV infection with kidney diseases is well known. ${ }^{8}$ Kidney involvement usually includes glomerular or vascular injury and expresses in many morphologic patterns. The histologic manifestations of HBV-associated kidney disease can be classified as those that occur as a result of either immune complexmediated glomerulonephritis such as MN, MPGN or IgA nephropathy or immune complex - related vasculitis 


\section{Chronic HBV \& RPGN}

such as polyarteritis nodosa (PAN). ${ }^{9} \mathrm{MN}$ is the most common pattern of glomerular disease detected, particularly in children.

It is important to note that many patients with chronic HBV infection may also harbor co-existent human immunodeficiency virus (HIV) $(5 \%-10 \%)$ and HCV $(10 \%-30 \%)$ that may dictate the glomerular pathology expressed and further broaden the clinical differential diagnosis. Many studies have found a significantly higher carrier rate of HBsAg in MPGN in both children and adults. As in HBV-MN, patients with MPGN often do not have a history of clinical hepatitis despite having abnormal liver function tests. Serum complement levels (C3, C4) are often low. We could not perform the staining for HBsAg or HBeAg on our kidney biopsy specimen due to non-availability of these tests. Thus, we have no direct evidence of HBV involvement in this immune complexmediated glomerulopathy, but the clinical scenario with which the patient presented and prompt response to antiviral therapy and kidney remission in conjunction with virological remission in the backdrop of well-known association of HBV infection with MPGN, we labelled this case as MPGN secondary to HBV infection.

HBV-GN rarely presents as RPGN. Although focal crescents may be seen on kidney biopsy in any form of MPGN, presence of $>50 \%$ crescents (crescentic MPGN) is an infrequent presentation and very few cases have been reported in literature. Lai et al. reported two cases of chronic hepatitis B carriers presenting with crescentic glomerulonephritis. ${ }^{10}$ One of them was diagnosed with crescentic glomerulonephritis with endocapillary proliferation who had a spontaneous remission despite persistent hepatitis B surface antigenemia. The second patient, who was diagnosed with MN on first biopsy and responded to symptomatic therapy, later presented with recurrence of proteinuria and kidney failure and the second biopsy revealed crescentic glomerulonephritis along with MN and MPGN pattern. ${ }^{10}$ This patient presented with nephrotic syndrome and acute kidney failure with crescentic MPGN on kidney biopsy. He was treated with entecavir $(0.5 \mathrm{mg} / \mathrm{day})$ for 28 weeks, three doses of intravenous pulse methylprednisolone (500 mg) daily followed by oral prednisolone starting at $1 \mathrm{mg} / \mathrm{kg} / \mathrm{day}$, and 14 sessions of plasma exchange for a duration of seven months. The patient achieved complete kidney, hepatic and virological remission.

No studies have been reported regarding the treatment of HBV-MPGN. Nasri et al. reported a case of crescentic transformation of HBV-associated MPGN with high HBV DNA load. ${ }^{11}$ Their patient achieved remission with steroids and lamivudine treatment. Mareddy et al. also presented a case of 44-year-old man with chronic HBV infection do presented with rapidly progressive kidney failure and nephrotic syndrome and was found to have crescentic MPGN on kidney biopsy. ${ }^{12}$ They also treated the patient with multimodal therapy including steroids, antivirals and plasmapheresis and succeeded in achieving kidney and virological remission. In the present case, because the patient presented with RPGN and kidney biopsy showing crescentic MPGN, he was managed with intravenous methylprednisolone along with entecavir and plasma exchange to reduce the immune complex deposits. He was not treated with more potent cytotoxic drugs like cyclophosphamide because of high viral load, which could have led to an acceleration of viral replication and increase in viral load with possible detrimental consequences. Insufficient evidence is available to confirm whether antiviral therapy against HBV infection improves the kidney prognosis of HBV-GN, and further well-designed prospective studies are needed.

\section{Conclusion:}

This case highlights a rare kidney manifestation of HBV infection requiring a high degree of suspicion and an early kidney biopsy for prompt diagnosis. It also underscores the need for a multidisciplinary and aggressive but balanced treatment approach (plasmapheresis and immunosuppression along with anti-viral therapy) for better clinical outcomes.

\section{Conflict of interest}

The authors declare that they have no conflict of interest.

\section{Ethical approval}

All procedures performed in this case report were in accordance with the ethical standards of the institutional committee and with the 1964 Helsinki Declaration and its later amendments or comparable ethical standards. 


\section{Chronic HBV \& RPGN}

\section{Informed consent}

Written informed consent was obtained from the patient for publication of this case report and any accompanying images.

\section{References:}

1. MacLachlan JH, Cowie BC. Hepatitis B virus epidemiology. Cold Spring Harb Perspect Med. 2015;5(5):a021410. doi: 10.1101/cshperspect.a021410.

2. Iloeje UH, Yang HI, Chen CJ. Natural history of chronic hepatitis B: what exactly has REVEAL revealed? Liver Int. 2012 Oct;32(9):1333-41. doi: 10.1111/j.1478-3231.2012.02805.x.

3. Ali M, Idrees M, Ali L, Hussain A, Ur Rehman I, Saleem S, Afzal S, Butt S. Hepatitis B virus in Pakistan: a systematic review of prevalence, risk factors, awareness status and genotypes. Virol J. 2011 Mar 6;8:102. doi: 10.1186/1743-422X-8102.

4. Lee H. W., Chon C. Y., Park Y. N., et al. Histopathologic correlation between chronic hepatitis B and nephropathy. Korean J Hepatol. 2001; 7:413 - 422.

5. Bhimma R., Coovadia H. M. Hepatitis B virus-associated nephropathy. Am J Nephrol. 2004; 24(2):198 - 211. doi: $10.1159 / 000077065$.

6. Yi Z., Jie Y. W., Nan Z. The efficacy of anti-viral therapy on hepatitis b virus-associated glomerulonephritis: a systematic review and meta-analysis. Ann Hepatol. 2011; 10(2):165 - 173.

7. Fabrizi F., Dixit V., Martin P. Meta-analysis: anti-viral therapy of hepatitis B virus-associated glomerulonephritis. Aliment Pharmacol Therap. 2006; 24(5):781 - 788. doi: 10.1111/j.1365-2036.2006.03041.x.

8. Gupta A, Quigg RJ. Glomerular diseases associated with Hepatitis B and C. Adv Chronic Kidney Dis. 2015;22(5):343-51.

9. Venkataseshan VS, Lieberman K, Kim DU, Thung SN, Dikman S, D'Agati V, Susin M, Valderrama E, Gauthier B, Prakash A, et al. Hepatitis-B-associated glomerulonephritis: pathology, pathogenesis, and clinical course. Medicine (Baltimore). 1990 Jul;69(4):200-16.

10. Lai FM, Li PK, Suen MW, Lui SF, Lai KN. Crescentic glomerulonephritis related to HB virus. Mod Pathol. 1992;5(3):262-7.

11. Nasri H, Mubarak M. Sudden deterioration of kidney function in a patient with nephrotic syndrome and a very high hepatitis B viral DNA load. J Kidney In Prev. 2012; 1(1):39 - 41.

12. Mareddy AS, Rangaswamy D, Vankalakunti M, Attur RP, Nagaraju SP, Koti N. Immune mediated crescentic MPGN secondary to HBV infection: A rare presentation for a common infection. AMJ 2016;9(1): 12 - 16. 